\title{
Catherine DELCROIX, Ombres et lumières de la famille Nour. Comment certains résistent face à la précarité
}

\section{Gabrielle Varro}

\section{(2) OpenEdition}

1 Journals

Édition électronique

URL : https://journals.openedition.org/remi/4146

DOI : $10.4000 /$ remi.4146

ISSN : $1777-5418$

Éditeur

Université de Poitiers

\section{Édition imprimée}

Date de publication : 30 juin 2007

Pagination : 181-182

ISBN : 978-2-911627-44-6

ISSN : 0765-0752

\section{Référence électronique}

Gabrielle Varro, "Catherine DELCROIX, Ombres et lumières de la famille Nour. Comment certains résistent face à la précarité ", Revue européenne des migrations internationales [En ligne], vol. $23-n^{\circ} 1$ | 2007, mis en ligne le 27 novembre 2008, consulté le 14 avril 2022. URL : http://journals.openedition.org/remi/ 4146 ; DOI : https://doi.org/10.4000/remi.4146

Ce document a été généré automatiquement le 14 avril 2022.

(c) Université de Poitiers 


\title{
Catherine DELCROIX, Ombres et lumières de la famille Nour. Comment certains résistent face à la précarité
}

\author{
Gabrielle Varro
}

\section{RÉFÉRENCE}

Catherine DELCROIX, Ombres et lumières de la famille Nour. Comment certains résistent face à

la précarité, Paris, Petite bibliothèque Payot, 2005, 258 p., ISBN 2-228-89963-1

1 Réédité en "poche », ce livre, publié une première fois en 2001, retrace l'expérience d'une famille berbère d'origine marocaine en France, famille réelle mais transformée en allégorie, afin de servir en quelque sorte d'exemple - comme son sous-titre l'indique - pour tous ceux qui sont confrontés à la précarité. Il ne s'agit pas de l'histoire collective d'un groupe d'immigrants mais de la biographie d'une famille, dont les dix membres deviennent emblématiques de «ceux qui, comme les Nour, se débattent face à l'adversité et veulent s'en sortir, telle est l'ambition de ce livre » (p. 23).

Une fois admise la posture militante de l'auteur, qu'elle assume d'ailleurs entièrement, et un style un peu surchargé d'adjectifs, le lecteur trouvera non seulement une mine de renseignements concernant les définitions, les effets sociaux et politiques de la pauvreté et de la précarité sur une famille et ses membres individuels, mais également des données concrètes, qu'elle a pu recueillir « depuis une vingtaine d'années en France, en Belgique, en Algérie et en Égypte, parce qu['elle faisait] comprendre aux ménages concernés $q u[' e l l e]$ ne venai[t] pas pour les interroger, puis les présenter comme des parents démissionnaires, mais pour comprendre les efforts qu'ils faisaient pour surmonter leurs difficultés et celles de leurs enfants » (18).

D’entrée de jeu, C. Delcroix « répond » ainsi aux préventions et aux craintes du citoyen que les médias dépeignent comme inquiété par l'image de "paresseux» et de «profiteurs » que certains politiciens répandent au sujet des populations migrantes. 
Parmi d'autres données concrètes contenues dans l'ouvrage, on relève un budget familial détaillé (104 sq.), des lettres de psychiatre (122-123) ou de procureur (126), de nombreux extraits d'entretiens... Se réclamant d'une approche ethnosociologique, C. Delcroix réussit ainsi à mêler à son expérience de sociologue auprès des familles enquêtées et aux données qu'elle a personnellement recueillies sur le terrain, de nombreuses informations et analyses provenant d'autres spécialistes (sociologues, économistes, démographes, historiens..), convoqués sur l'immigration et les conditions de vie des classes populaires en France.

De cette manière, l'auteur nous donne et donne aux personnes concernées au premier chef - les Nour et leurs semblables, ainsi que les acteurs sociaux à qui ils ont affaire à la fois une vision objective et distancée des réalités sociales et politiques qui conditionnent leur existence et une compréhension des effets subjectifs qui en découlent. Dans la première partie du livre, s'agissant « des enfances ", tant des parents avant leur émigration que de leurs huit enfants nés en France, on voit la différence des générations dans la fratrie: les aînés investis dans la réussite scolaire, mus par la volonté de ne pas décevoir les espoirs de leurs parents et les petits qui n'ont plus « peur de rien » et sont « plus difficiles à éduquer » (58).

6 L'aperçu que nous avons des acteurs sociaux rencontrés par $\mathrm{M}^{\mathrm{me}}$ Nour et ses enfants (assistantes sociales, orthophonistes, psychologues, animateurs, infirmières, professeurs, médecins, éducateurs, etc.) correspond à l'expérience que, face aux diverses administrations, ce type de famille peut avoir tous les jours (sans exclure d'ailleurs les exemples de générosité et de solidarité de la part de certains de ces acteurs). L'ouvrage de C. Delcroix en devient un manuel à double titre : à l'usage des familles, surtout des mères - et si elles sont illettrées, leurs enfants le leur liront ! soucieuses du devenir de leurs enfants; mais aussi à l'usage du public français plus généralement et des professionnels en particulier. Grâce à ce livre, en effet, ceux-ci verront ce que leurs actions risquent parfois d'avoir de trop « contrôlant » ou de trop "systématique ». Ils trouveront également une présentation sensible et complexifiée de vécus que parfois ils sont loin d'imaginer, dont un des exemples les plus frappants est probablement l'expérience de bigamie de son époux que $\mathrm{M}^{\mathrm{me}}$ Nour a décidé d'accepter, pour que ses enfants "continuent à vivre avec leur père, qu'il ne les laisse pas» (100 sq.).

7 On peut regretter que l'auteur ne propose pas d'explication à tous les phénomènes et circonstances qu'elle décrit : par exemple, concernant « la langue qu'il est bon de parler avec sa fille ou son fils» (73), elle aurait pu préciser que le conseil de parler en français avec leurs enfants, que beaucoup de parents allophones reçoivent de la part des enseignants ou de psychologues, ne fait que refléter une frilosité ethnocentrée par rapport au bilinguisme, attitude courante dans le système scolaire français (comme le rappelle le numéro 116 de la revue Langage et Société, 2006). Fort heureusement, $\mathrm{M}^{\mathrm{me}}$ Nour "n'a pas suivi les conseils des professionnels » et emploie le berbère et le français, "pour qu'ils puissent parler avec leurs grands-parents", et elle a bien raison. Toutefois, il y a aussi dans l'ouvrage de C. Delcroix des facteurs finement analysés, notamment - à travers l'étude d'un des fils Nour qui s'est retrouvé en prison - le sentiment récurrent de l'injustice, effet parfois d'une seule phrase de mépris ou de rejet de la part d'un enseignant ou d'un éducateur, mais qui risque même de devenir, chez des jeunes susceptibles, un premier pas vers la délinquance. 
8 Livre vivant, qui a permis - d'après la lettre de la fille aînée qui clôt le volume - à ces protagonistes exemplaires de voir les événements de leur vie transformés en récit (215), les observations de C. Delcroix, adossées à un grand nombre de références savantes et résumées dans une Postface rétrospective, donnent à voir les stratégies que des gens qui cherchent à survivre et à réussir dans une société où ils sont "discrédités» mettent en œuvre, quand ils font preuve, comme la famille Nour, d'humour, de courage et d'ouverture aux autres.

\section{AUTEURS}

\section{GABRIELLE VARRO}

Laboratoire Printemps, Université de Versailles Saint-Quentin 